\title{
Germanica
}

\section{Dramaturgien der Verspiegelung (en). Ein Streifzug durch die deutsche Gegenwartsdramatik}

Dramaturgie de la (des) "réflexions déformantes ». Le théâtre allemand d'aujourd'hui

\section{Stefan Tigges}

\section{OpenEdition}

\section{Journals}

Édition électronique

URL : http://journals.openedition.org/germanica/455

DOI : $10.4000 /$ germanica. 455

ISSN : 2107-0784

\section{Éditeur}

Université de Lille

\section{Édition imprimée}

Date de publication : 1 décembre 2005

Pagination : 121-133

ISBN : 2-913857-16-7

ISSN : 0984-2632

\section{Référence électronique}

Stefan Tigges, « Dramaturgien der Verspiegelung (en). Ein Streifzug durch die deutsche Gegenwartsdramatik », Germanica [Online], 37 | 2005, Online erschienen am: 07 Januar 2010, abgerufen am 06 Oktober 2020. URL : http://journals.openedition.org/germanica/455 ; DOI : https:// doi.org/10.4000/germanica.455

Ce document a été généré automatiquement le 6 octobre 2020.

(c) Tous droits réservés 


\title{
Dramaturgien der Verspiegelung (en). Ein Streifzug durch die deutsche Gegenwartsdramatik
}

\author{
Dramaturgie de la (des) "réflexions déformantes". Le théâtre allemand \\ d'aujourd'hui
}

Stefan Tigges

Geschichte kommt als Störung vor, wie Mücken

im Sommer.

Heiner Müller in einem Brief an Pina Bausch

1 Wir dramatisieren von Natur aus, schlüpfen je nach Lebenskontext in die verschiedensten Rollen, setzen uns in Szene und leben zunehmend mit und in den sich in unserer Gesellschaft ausbreitenden Inszenierungs-Strategien. Das Drama Leben bildet in all seiner Theatralität und großen und kleinen Spektakularität seit der Geburtsstunde des Theaters die Vorlage für das, was Dramatiker dramatisch verdichten und uns schließlich als Spiegel vor Augen halten : Eine Geschichte unserer Geschichte, tragisch oder komisch erhöht, weniger visionär als unsere Befindlichkeiten in zeitlicher Verzögerung theatralisch nachzeichnend, überschreibend und abbildend. Die von den Dramatikern eingefangenen schönen und hässlichen Bilder und Bildlichkeiten unserer kulturellen, sozialen, politischen und ökonomischen Lebenspraxis werden im Verlauf ihres Übersetzungsprozesses ästhetisch mehrfach gebrochen und entwickeln schließlich durch den Abstand zum Leben und das geleistete Abstraktionsvermögen eine künstlerische Sprache, die sich dramatisch und im Blick auf die postdramatische Aufführungspraxis wachsend undramatisch äußert.

2 Im Folgenden soll in Form von Lektüren unterschiedlicher Theatertexte zweier Autoren, deren Dramaturgien auf ihre Spiegelungs- und Verspiegelungsfunktionen beleuchtet werden, wobei unter Verspiegelungen eine Stufe verstanden wird, die sich durch divergierende Strategien von "einfacheren " Realismuskonzeptionen der Spiegelung absetzt. Die These dazu lautet, dass in den ver-zerrten Bildwelten der Verspiegelungen im Gegensatz $\mathrm{zu}$ den gespiegelten Bildwelten des dramatischen 
corpus die polarisierende Gegenüberstellung von «Schönem » und "Hässlichem » durch eine Strategie des Verwischens an Bedeutung verliert.

Es scheint sich in Analogie zu unserer Gesellschaft, die ihre hässlichen Züge ästhetisch überblendet und um- inszeniert, ein werkimmanentes Spiel der Versetzungen und Zersetzungen von «Schönem » und «Hässlichen » durchzusetzen, die unmittelbar aneinander gekoppelt sind und deren Trennungslinien sich in ihrer Werk-Ästhetik verlieren.

4 Mit dem Spiel der Unterscheidungen soll aber keineswegs eine weitere DramenKategorisierung vorangetrieben als vielmehr auf dramenästhetische Tendenzen hingewiesen werden, die in immer größerer Simultaneität im neuen Dramentypus auftreten.

5 Unter dem Moment des "Schönen" möchte ich primär ein künstlerisches dramatisches Ausdrucksvermögen verstehen, das in idealistischer Art und Weise nach neuen gesellschaftlichen utopistischen Partikeln sucht, humanistische und moralische Restgrundwerte möglicherweise in hoffnungsvoller Manier neu verhandelt und sich durch ein träumerisches und phantastisches Ausdruckspotential der dramatis personae auszeichnet, die sich schließlich im Inhalt, der Form und vor allem im (poetischen) Sprachmilieu des Werkes ausdrücken.

6 Zum « Hässlichen » ließe sich eine ideologieverseuchte, identitätsgestörte Dramenwelt der Gewalt und der absoluten ökonomischen Hegemonie zählen, in der eine Vielzahl von Figuren als Opfer dramatisch zwischen einer « Ästhetik des Schreckens » und einer « Ästhetik der Katastrophe » umherirren und sich vor allem auf den zynischen Kriegsschauplätzen der jeweils auseinander brechenden Familien- und Arbeitswelt sprachlich und körperlich in einer spezifischen Rohheit attackieren und dabei die allerletzten Tabus berühren, zählen.

\section{Die Poetik der Katastrophe : M Rinkes (gestörtes) romantisch-dramatisches Basteln an einer schönen neuen Welt}

7 «Wie viel Weltmoral brauchen wir für das 21. Jahrhundert? Was ist überhaupt heute Moral ? Wollen wir sie? Und wenn wir sie wollen, wie machen wir es, dass auch andere sie wollen?" fragt einer von Rinkes unerschütterlichen und fast unbeirrbaren Weltverbesserern in einem Stück, das auch noch Die Optimisten heißt.

Rinke stattet, wie kaum ein anderer Autor im deutschsprachigen Raum, seine dramatischen Sprachrohre mit einem aberwitzigen sozial-utopistischen Sprachgestus aus und siedelt seine Stücke, die sich ohne weiteres als idealistische Modellversuche von Gesellschaft lesen lassen, in phantasievollen Zwischenwelten an, welche sich durch ihre charakteristische Exotik von den allgemeinen gesellschaftlichen Grauzonen und dramatischen Mustern abheben.

9 Entgegen verschiedener postdramatischer ästhetischer Transforma-tionsformen vertraut Rinke auf eine fast konventionelle dramaturgische Kompositionsweise, die trotz einiger Brechungen auf mehr oder weniger psychologisierte Figuren setzt, denen der Autor Text zuordnet und eine bildreiche Sprache in den Mund legt, die dann aus der geschaffenen Situation heraus die ausgedünnten Handlungsstränge bewegt. 

heruntergekommenen Gebäuden auf, an denen die Zeit genagt hat und die von der Außenwelt abgeschnitten sind. In auffälliger Gesellschaftsferne tüfteln Rinkes Protagonisten dann während ihrer Zusammenkunft in aller Abgeschlossenheit an gesellschaftlichen Utopien, um für sich und die Außenwelt träumerisch neue Lebensräume zu -öffnen.

11 In Republik Vineta (2000) wählt Rinke als Schauplatz ein ehemaliges Sanatorium auf einer kleinen Ostsee-Insel, in dem eine Gruppe von gestrandeten Spezialisten in einem etwa fünfwöchigen Modellversuch eine neue Stadt-Republik erschaffen und damit Geschichte machen soll. Den Ort ihrer Zusammenkunft, an dem schon Napoleon 1812 seine Russlandinvasion plante, der im Verlauf der vier Akte immer mehr zusammenstürzt, skizziert Rinke in einer Szenenan-weisung im ersten Akt wie folgt: «Ein großer Raum. Halblinks eine Konferenztafel mit sechs Stühlen. Eine riesige Pinnwand mit Plänen, Skizzen, Seekarten, Zahlen. Dahinter weht leicht ein Vorhang, und es ist, als höre man draußen das Rauschen eines Waldes oder die Wellen eines Meeres. In der Mitte hinten eine offen stehende, große, renovierungsbedürftige Flügeltür. Daneben ein baufälliger Kamin mit Sessel. Irgendwo im Raum ein Kaffeeautomat. Hinten rechts eine große, schon etwas poröse Marmortreppe, die auf die Galerie führt... »

Die Projektteilnehmer, die sich aus Architekten, Ingenieuren, Stadtplanern, einem Kapitän, Politiker und Arbeitsamtbereichsleiter zusammensetzen, arbeiten vor Ort Tag und Nacht bis zur Erschöpfung mit- und gegeneinander an ihrem Vorhaben, dessen Planungsschritte sich abstrus vergrößern und immer wahnwitzigere Züge annehmen. Die am Ende unvollendete symbolische Vineta-Vision, die das Leben heller machen und Künstler, Wissenschaftler und Philosophen im Rahmen einer internationalen Weltakademie in einem Stadtviertel integrieren und statt « den Verkehr das Gespräch

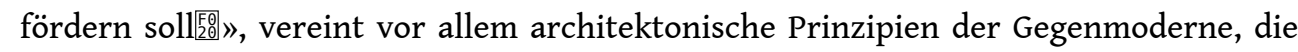
sich in ihrer Schöngeistigkeit und Sehnsucht nach Einheitlichkeit und Harmonie von den postmodernen Schäden abzusetzen versuchen.

Der Architekt Färber, womöglich ein Enkel von Frischs Faber, der Mehrzweckhallen hasst, füllt als Vertreter der Rückbesinnung sein Vineta mit Laboratorien, Auditorien, Ateliers und Bibliotheken, die er von altrömischen Bauten, griechischen Tempeln und toskanischen Säulen umgeben sieht. Sein Kollege Montag vom Arbeitsamt, der im zukünftigen Vineta für die Einstellungen verantwortlich ist, träumt von einer «Fakultät der Empfindung », der Politiker Behrens von « Natur trotz Globalisierung » und « Biotopvernetzung ».

14 Der Projektstrukturplaner Hagemann glaubt dagegen an seine Parole « Sein ist Tun und Tun ist Tempo » und setzt auf eine Stadt-Vision, die sich aus Cinemaxx, Club Med, Wellness, Wohnblöcken und Verkehrsströmen zusammensetzt. Zwischen die Fronten platziert Rinke schließlich den vermittelnden Projektleiter Leonhard, der einen Themenpark konzipiert, der die alte Welt als "Die untergegangenen Träume» ausstellt, wozu auch eine überdimensionale Lenin-Statue gehört, jedoch dafür als Kompromiss moderne architektonische Ummantelungen fordert.

Inmitten der Euphorie Färbers, der der Welt die verloren gegangene Harmonie zurückgeben möchte und vergessene öffentliche Plätze, Gassen und Strassen als «musikalische Pausen » in seinem Entwurf komponiert, vergiftet sich das Klima unter den überarbeiteten Weltverbesserern durch Putschversuche und Pistolenduelle 
zunehmend, wodurch das idealisierte schöne kollektive Bauen an einer neuen Welt erste hässliche Risse bekommt.

Im dritten Akt lüftet Rinke schließlich das Geheimnis, nachdem Leonhard den Unfrieden der Beteiligten, die jedoch verbissen an ihren Arbeiten festhalten, auch nicht mit seinem Programm des gemeinsamen Musizierens, Erdepflügens und Heuriechens beseitigen konnte: Das Projekt Republik Vineta ist nichts als ein Versuch, die reale Arbeitswelt zu simulieren und die Teilnehmer durch den anschließend herbei gefügten schockartigen Aufprall auf die Realität auf ein Leben ohne oder nach der Arbeit vorzubereiten und sie zu lehren, «ihre Träume in Würde zu begraben», wie es Rinkes Projektleiter formuliert.

Im letzten Akt wird das böse Scheitern des Projektes immer offensichtlicher. Statt des gewünschten Realitätsaufpralls verfransen sich die Experten zunehmend in ihren absurd-schönen Planungsvisionen einer neuen Welt ohne dabei zu bemerken, wie sie dabei auf der Strecke bleiben und sich währenddessen noch gegenseitig aus dem Weg schaffen.

Rinkes komisches Nachspiel des vielleicht längst verlorenen gesellschaftlichen Endspiels endet im Fiasko, die Welt ist keineswegs heller geworden, dafür aber einmal wieder schön dramatisch auf den Kopf gestellt und politische, soziologische und urbanistische Diskurse spielerisch bewegt worden. Ein Sprachballett von untoten Mittelstandsleichen, die gegen den Zeitgeist revoltieren und diesen zugleich dramatisch verspiegeln...

In Die Optimisten (2003) versammelt Rinke erneut eine bunt gemischte Gruppe, die er dieses Mal aus dem Bildungsbürgertum rekrutiert, an einem von der Zivilisation entfernten exotischen Ort. Eine Reisegruppe, zu der u.a. eine Hörfunk-Journalistin, eine Studentin, eine Medizinerin von "Ärzte ohne Grenzen », ein angehender Filmregisseur und ein Ministerialrat aus dem Bildungsministerium gehören, bleibt während ihrer Bildungsreise für eine Woche in einem verlassenen Hotel in Lumbini/Nepal stecken. Die Reiseroute, die Die Optimisten noch in einen Nationalpark, nach Kathmandu und schließlich nach Bombay führen sollte, wo eine internationale Konferenz zur Agrarund Wirtschaftspolitik stattfindet, wird von Rinke jäh gestört, indem die missionarische Wallfahrt zunächst durch eine Buspanne unterbrochen wird und schließlich ein maoistischer Aufstand bis an das Hotel vorrückt, in dem die Gruppe allein eingesperrt ist. Der Kontakt zur Außenwelt bricht ab. Der Wirtschaftsethiker Thomas Matt, der in Bombay eine Petition für eine humanere und solidarischere Welt und für die Entschuldung der dritten Welt überreichen und die Bildungsurlauber auf die Konferenz einstimmen sollte, erreicht das leer stehende Hotel nur noch als Leiche. Der Busfahrer, der sich auf die Suche nach Ersatzteilen gemacht hatte, verschwindet ebenso mysteriös wie später der Reiseleiter.

Die Eingesperrten vertreiben sich zunächst mit esoterischen Ausflüchten, Meditationsübungen à la "Das Erreichen der inneren Meeresstille », Marx-Lektüren und dem Verfassen einer eigenen Petition die Zeit, deren Fluss immer stärker aufgehoben wird. Die täglichen und nächtlichen Hotellobbydiskurse bei Erdnüssen und den Getränken aus den Minibars über "Solidarität und Utopien im 21. Jahrhundert » werden zunehmend von den Geschehnissen aus der Außenwelt überschattet, die sich für die Beteiligten jedoch nur als dunkle Ahnung äußern. Die Optimisten verbleiben in ihrer Welt, werden nicht zu Realisten und entwickeln jeweils lustvoll ihre Gesellschaftsvisionen. 
21 Während der Filmemacher Nick an einem neuen Projekt spinnt - «Die Verträge, die Abkommen liegen zur Unterschrift bereit, ein Hai lässt sich mit seinem Montblanc in den Sessel fallen. Er will gerade seine Unterschrift setzen unter ein Staudamm-Projekt, das wieder Hunderttausende ins Verderben stürzt, und plötzlich verwandelt sich das Staudamm-Projekt in eine Taube und fliegt mit seinem Montblanc weg zur Globalisierungskonferenz nach Bombay. Ich meine, so ne Art surrealistischer Film : Neue Angriffslust gepaart mit angeschrägter Poesie, Taube ist vielleicht zu Amnesty International, einfach sone ganz normale Möwe... »-, plant die Hörfunk-Journalistin Inken Hellinger einen dokumentarischen Vorort-Beitrag für ihre Sendung «Von Sonnenstrahlen und Sonnenmenschen ».

Die idealistische Studentin Carla verurteilt in ihrer verfassten Resolution das amerikanische "Terminator-Gen », das die indischen Reis- und Zuckerrohrbauern in den wirtschaftlichen Ruin treibt. Unterdessen leitet die Ärztin Maria Berger unter dem Motto «Buddha für Gestresste » weitere fernöstliche Philosophiekurse und verarztet kleinere «Zwischenfälle ». Ministerialrat Kraus konzentriert sich auf politischwirtschaftliche Dimensionen und philosophiert als Alt-68er ähnlich wie Leonhard über Max Weber in Republik Vineta über die Mehrwert-Theorie von Marx, und versucht den "Urmechanismus der Ausbeutung " theoretisch $\mathrm{zu}$ bremsen und diagnostiziert schließlich resignierend eine gesellschaftsformunabhängige Akkumulation : «Akkumulation, Carla, ist aus der Welt nicht wegzukriegen. Wir proletarisieren ganze Erdteile. Wir grasen die nicht-kapitalistischen Länder ab nach dem, was wir brauchen können. Wir machen sie kapitalistisch, aber nur um besser grasen zu können, und dann, wenn alles abgegrast ist, ziehen wir weiter und lassen eine Hülse zurück, die weder sozialistisch, kommunistisch, kapitalistisch noch sonst was ist. ”

23 Nick verurteilt Krauses 68-er Haltung der « soziopolitischen Wir-Bindung » und spinnt ein medial aufgerüstetes Konzept, das entfernt an die Vineta- Konzeption der « internationalen Weltakademie » erinnert : «Ich spreche von neuer Moral.... Moral geht nur noch über die mediale Mikro-Schiene. Über den kommerzialisierten Nahbereich geht sie dann über in die politische Makro-Ebene. Sie wollen da immer mit ihrer Pflichtethik, aber das geht nicht. Ich rede nicht von Turnschuhen oder Schlaghosen, sondern mir schwebt da etwas vor wie wirkliche Werteagenturen... »

Am Ende des Stückes sind die Erdnuss- und Minibarvorräte fast aufgebraucht. Die Eingeschlossenen kauern, wie die Tiere draußen hinter der zersplitterten Glasfassade auf der Terrasse, auf ihren Matratzen zusammen gepfercht auf dem Boden der Hotellobby. Die Unruhen und die « Animalität » sind von der Außen- in die Innenwelt vorgedrungen und von den Insassen adaptiert worden. Kraus hat Nick erschossen und Inken spricht in völliger Dunkelheit bei Brahms-Musik aus dem Kassettenrekorder einen Beitrag auf Marias Diktiergerät und erinnert dabei sehr an die träumenden Protagonistinnen aus Cechovs Dramen: "...hier ist Antenne sieben... ich hoffe, die nächsten hundert, tausend Jahre werden ein paar solcher Tage haben. Solche Tage sind endlich hell, ohne sie geht es nicht, den Tagen der Sonnenmenschen... »

Trotz des Zusammenfalls der beiden Welten und der Bruchlandung der verbliebenen Optimisten sendet Rinke damit aus der erweiterten Kampfzone ein hoffnungsvolles Signal, dessen utopischer Gehalt eine «schönere » Welt, ungeachtet ihrer hässlichen Auswüchse von Globalisierung und Terrorismus, für möglich hält.

In Café Umberto (2005) verfolgt der Autor eine andere Strategie, holt die Außenunmittelbar in die Innenwelt und platziert seine dramatis personae an einem 
Schauplatz, der in unserer heutigen Zivilisation regen Zulauf erfährt : « Der Saal eines Amtes. Eigentlich für ein Arbeitsamt ein schöner Saal, nur ziemlich heruntergekommen, die Kommune ist vermutlich in die Krise geraten. Eine riesige Tür, darüber diese klassische Nummernanzeige... Es gibt auch den Nummernau-tomaten, Sitzbänke, Bilder, ein großes Fenster, vielleicht eine Fahne -irgendwo, den Block einer staatsmännischen Büste und einen einfachen Coffee-Shop, der noch geschlossen ist... »

Die Agentur für Arbeit steht irgendwo in Berlin und wird im Verlauf des Stückes von den Wartenden, die über viel Zeit verfügen, von Innen heraus transformiert, was bei Rinke einer komisch überzeichneten Minirevolution gleichkommt. Hier treffen in 18 Bildern, die sich auf einen nicht festgelegten Zeitraum erstrecken, Arbeit suchende Künstler, Akademiker, Modedesigner und ein Landwirt auf den Italiener Umberto, der vor den Bürotüren erfolgreich eine Ich-AG gegründet hat und die Wartenden mit dem besten Berliner Latte Macciato beglückt. Statt kollektiver Depression, Vergangenheitsnostalgie, Zukunftspanik oder aufkommender Konkurrenz bei der Jagd nach einer Tätigkeit, wobei die Angebote nur äußerst selten per Stimme aus einem Automaten dringen, gerät die Zusammenkunft trotz zahlreicher individueller Rückschläge und Erniedrigungen aller Beteiligten erstaunlich lebensfroh.

Der Flurbereich des Amtes wird für die Modedesignerin Jule, die zuvor ihre Kollektion «Berlin am Meer » aus ihrer Wohnung heraus an einer Schnur über einer Hauptstrasse präsentiert hatte, zeitweilig zum Laufsteg, einer wenn auch verunglückten neuen Präsentation. Umberto, die heimliche aber stumme Seele des Amtflures, kehrt mit der Asche seines verstorbenen Vaters aus Italien zurück und erfährt bei der Renovierung seiner Kaffeebude, die auch einen Altar für die Urne vorsieht, große Hilfe, was dazu führt, dass die gezogene Registriernummer aus dem Automaten auch schon einmal weiterreicht und in aller Gemütlichkeit noch einen weiteren Milchkaffee getrunken wird, um dann nach Hause $\mathrm{zu}$ gehen. Statt ernüchternder, eisiger und desillusionierender Atmosphäre entsteht nach einem Anstrich und sparsamer Möblierung ein suggeriertes "Wohlempfinden", das den umschulungs-müden Erdkundelehrer Lukas, den Mann der sprechenden Stimme aus dem Automaten, die von einer berühmten Fernsehmoderatorin zur Verfügung gestellt wurde, dazu verleitet, sich direkt vor Ort einzurichten und dabei zugleich nahe der Stimme seiner Frau zu sein, die viel zu viel arbeitet und deren Stimme er zu Hause nur selten hört. Lukas diagnostiziert bei sich einen Verlust von Leichtigkeit, fühlt den « aufziehenden Frost in seinen Gliedern » und sucht nach einem Ausweg: "Das zu begreifen, dass nichts mehr vor einem liegt ! Ich glaube, das ist die erste Nachricht, die man bekommt von seinem eigenen Tod. Plötzlich nimmt einen diese Ahnung an die Hand und zeigt uns, wie das Schiff sich langsam dreht. Die ganze Lebenskraft auf offener See zurück... Ich mache mir selber therapeutische Projekte... »

Durch Gesprächsfetzen und Blenden, die aus dem Amt heraus führen, verdichten sich die Bilder über die tragisch-komischen abschüssigen Wege der Betroffenen, die temporäre Nebentätigkeiten als Haupttätigkeiten ausführen oder durch groteske Umschulungs-Maßnahmen durchs Leben schlittern. Die gestörten, unterbrochenen und umwegreichen Lebensläufe werden von Rinke dramatisch ver-rückt und können wie bei Jule, die später erfolglos einen « Ozonerzeuger » zu vertreiben versucht, auch in der Psychiatrie enden.

30 Anton, ein arbeitsloser Akademiker, dessen Frust sich schließlich auch im Intimverkehr mit Paula niederschlägt, gründet mit seiner Frau, die einmal Balletttänzerin war, 
zusammen einen «Laubsauger-Vertrieb" und verunglückt letztlich tödlich als Streckenposten bei der Bahn, indem er statt eines Warnschildes eine Tafel mit der Aufschrift « Heute keine Vorlesung » hochgehalten hatte.

31 Es gibt aber auch hoffungsvollere Modelle wie z.B. den Musiker Jaro, der während seiner Tätigkeit als Friseur an seiner Komposition der "Kopfschwingungen" weiterkomponiert und an einem großen Werk arbeitet, das grundmotivisch « wie ein Zug durchs leben fährt ».

Bei den Renovierungsarbeiten skandiert er als Wortführer der neuen glücklichen Arbeitslosen von der Leiter aus die neue Richtung: «Der Kampf um Erfolg, Erwerb, der da draußen wütet wie die Pest, er wird alles umbringen, was sich nicht radikal davon trennt! Ist ja nicht unbedingt neu, die Erkenntnis, muss man aber auch mal anwenden !... Wenn ich mich in diesem Land so umgucke, sehe ich überall Begabung, also, wir sind hier alle große Begabungen! Hier drin, da ist auch Arbeit in uns! Man darf sie halt nur nicht draußen zählen lassen! Die Hervorbringung der neuen Gesellschaft liegt nicht mehr im Zählen, die alte zählende Welt da unten befindet sich im Todeskampf! Ich fordere die Neue Arbeit und die Akademie für neue Selbsttätigkeit! Millionen können sich doch nicht einfach so stilllegen lassen !... Ich gründe die Akademie für Selbstachtung !... Wer da drin war, in der Akademie, holt sich die Neue Arbeit hier automatisch raus, die Gesellschaft geht nur so, wenn jetzt erst mal Würde kommt und dann irgendwann meinetwegen Erwerb, wir drehen das ab sofort um!»

Im weiteren Stückverlauf werden die optimistischen Parallelen zu Republik Vineta und Die Optimisten als auch der Glaube an eine neu aufkommende "Schönheit» der Gesellschaft immer deutlicher. Rinke führt in Form seiner fröhlich-komischen Kapitalismuskritik, die das System wieder auf den Kopf stellt, als zentralen Würdemotor erneut die Bedeutung und Veränderungskraft der Kunst an, lässt Jaro ein Vanmeer (Vermeer ?)-Gemälde an die Flurmauer des Amtes hängen und träumt von der heilenden Wirkung von Musik, Literatur und Drama.

In einem Gespräch mit Lukas wird zudem die Bedeutung der Zeit neu definiert. Statt die Zukunft vorweg zu nehmen oder die Vergangenheit zurück zu rufen, fordern beide sich wieder richtig in der Gegenwart aufzuhalten und dabei das "Zeit-Haben " in den Mittelpunkt zu stellen. Jaro : « ... Du musst, wenn du hier sitzt, einfach nur verstehen : Du verlierst nichts !... Wir müssen das Zeitverlieren überhaupt erst wieder lernen und zwar so lange, bis es kein Verlieren mehr ist, sonst brauchen wir auch gar nicht mit der Neuen Arbeit anzufangen! » Im Schlussbild hat sich die Welt nicht wirklich verändert: Jaro sitzt mit seinem Instrument im Amt und singt zusammen mit Umberto ein Lied von Paula, die er noch immer liebt und in einer Psychiatrie verschwunden ist. Verschwunden ist auch der Leiter des Arbeitsamtes, der selbst arbeitslos geworden ist.

\section{M von Mayenburgs Kriegsschauplätze der Familie und der Arbeit}

Für den jungen Dramatiker Marius von Mayenburg stellt sich das Bild der Gesellschaft wesentlich düsterer und hässlicher dar. Statt Visionen überwiegen in seinen Werken, die in ihrer Ästhetik stark durch die englische Dramatikerwelle der 90er Jahre um S 
Kane und M Ravenhill geprägt sind aber formal z.T. auch an die deutsche realistische Tradition des Familiendramas anknüpfen, nihilistische Grundtöne, die sich vor allem in der Vereinzelung seiner Protagonisten und in ihrer puren Sprachgewalt, die unmittelbar mit dem aggressiven Körpergestus korrespondiert und zusammen in « letzte» Tabus einbrechen, äußern.

Im Gegensatz zu Rinke, der «Schönes » mit « Hässlichem » versetzt, ist Mayenburgs dramaturgischer Kosmos, in dem das «Gute » nur ephemer durchblitzt, vom « Bösen » zersetzt.

In Feuergesicht (1998) zertrümmert Mayenburg die letzten rudimentären familiären Beziehungsstrukturen und spiegelt mit dem Bild der auseinanderklaffenden menschlichen Bindungen zugleich den Zustand der Gewalt, der sich in den heutigen Gesellschaftsformen immer prägnanter manifestiert. Im Verlauf der 94 dichten Szenen fokussiert der Autor zumeist aus der Perspektive von jugendlichen Pubertierenden insbesondere seinen Protagonisten Paul, der in einem blutigen Amoklauf seine Familie mordet, bevor er sich schließlich verbrennt.

Kurz vor seiner Selbstvernichtung resümiert der junge Titelheld : «Ihr macht euch alle eine falsche Vorstellung vom Leben. Der Mensch ist ein Gerät. Es wird ein Kraftstoff verbrannt, und es bewegt sich. Dabei entsteht Hitze. Solange einer noch verbrennt, lebt er. Ein Toter ist kalt, da brennt nichts mehr. Hitze, Feuer, das ist das Prinzip, das ist biologisch. Es ist nicht so, wie ihr denkt. Ihr seht euch nur im Verhältnis zu anderen, anders seht ihr nichts. Ihr wollt euch abspiegeln von den anderen und denkt, dass ihr damit was zu sehen kriegt von euch selbst, und dass ihr dann vorhanden seid. Was alles Mist ist. Ihr seht nur die andern. Ihr verschwindet. Ihr verdünnt euch, bis ihr weg seid, und wer die andern sind. Das ist der Fehler, dass ihr denkt, das muss so sein. Man muss sich rauskappen aus der Verbindung und einzeln werden, raus mit den fremden Gedanken und alles dicht machen, nach außen keine Fühler mehr, nur noch Waffen, wie eine Qualle, blind und zu, und wer sich nähert, wird verbrannt, ohne Wut. Mund zu, Ohren zu und tun!»

In den folgenden Stücken Parasiten (2000) und Das kalte Kind (2002) verbleibt der Autor im «Unterschichtmilieu » bzw. "Kleinbürgertum» noch im Mikro-Kosmos der «Familienhölle », in die jedoch langsam auch Außenstehende eindringen. Jenseits von sozialen Sicherheiten und menschlicher Wärme mutiert die Sprache seiner Figuren zu einem brutalen und kalten Folterinstrument, das die frustrierenden Lebensausschnitte immer wieder zusammenbrechen lässt. Auch hier steht die als animalischer Triebfaktor gezeichnete aufkommende Sexualität in unmittelbarer Nähe zu Gewalt und Perversion. Mayenburg verschraubt in seinen horrorartigen Minitragödien immer wieder gekonnt dunkle innere Monologe, montiert blutige Visionen mit Alpträumen und erreicht trotz des hässlichen Grundtimbres einen bildreichen Sprachwitz, der auch ins Komischskurrile kippen kann.

In Eldorado (2004) wird dann vom Autor zum ersten Mal wirklich der Familienkosmos geöffnet. Die zuvor nur als Hintergrundfläche angedeuteten hässlichen Schatten der Arbeitslosigkeit und des kriegerischen Zustands der Gesamtgesellschaft treten nun offen hervor. Das mögliche titelbezügliche Glücksversprechen ist bereits zu Anfang des Stückes im Keime erstickt.

Anton, beruflich in der von Krisen gebeutelten Immobilienbranche böse gescheitert, wird nach ausgiebigen Betrügereien von seinem Chef Aschenbrenner, der später in 
Konkurs geht und sich umbringt, entlassen und lebt seiner Frau, einer ausgebrannten Konzertpianistin, die von ihm ein Kind erwartet, ein erfolgreiches Berufsleben vor, bis alles auffliegt und mit seinem Selbstmord endet. Das geplante Haus wird damit nicht mehr fertig und die Familienstruktur vernichtet, bevor sie, wie in den vorherigen Stücken, drastisch auseinanderklaffen kann. Die Gesellschaft ist ein einziges Katastrophenszenario und befindet sich bis $\mathrm{zu}$ ihrer Auslöschung in einem permanenten Krieg. Aschenbrenner beschreibt deren Untergangsstadium in seinem Anfangsmonolog und zeigt damit, dass der Ästhetik des "Hässlichen » noch immer Züge des Poetischen und "Schönen» innewohnen: "Fern, hinter den dunklen Wäldern, sind Helikopter aufgestiegen, ein dunkler Schwarm, gestaffelt ausgebreitet ziehen sie herauf über der Stadt mit geschreddertem Dröhnen, taumeln über dem Gaswerk und neigen sich dann gegen den Bahnhof. Als kurz darauf die ersten Geschosse die Glaskonstruktion durchschlagen und einen Zug auf dem Gleis in voller Länge aufreißen, schneidet höher bereits ein Geschwader nachtschwarzer Kampfflugzeuge durch den roten Himmel. Es ist der Beginn der ersten Angriffswelle. Ein Vierteljahr später stehen wir vor einer einzigartigen Aufgabe. Das Areal, hat aus der Luft betrachtet, die Umrisse eines abgeschlagenen Kopfes. Nördlichster Punkt ist der Invalidenfriedhof, der sich mit dem abstrakten Weiß seiner umgesunkenen Grabsteine klar von den Trümmern der Umgebung abhebt. Vom westlichen Rand zieht der Geruch von verbranntem Tropenhölzern herüber, hier ist der botanische Garten bis ans Flussufer weggebrannt, das Orchideenhaus ist in der Hitze zerplatzt, die Tiere haben den angrenzenden Zoo prophylaktisch verlassen. Jetzt irren sie durch die Ruinen des Regierungsviertels und trinken aus den Springbrunnen, die jemand vergessen hat, aber das tut nichts zur Sache. Südgrenze ist das Sportstadion, das allerdings in seiner jetzigen Form erhalten bleiben soll. Hier klingen, wenn der Wind richtig steht, die Stimmen der Flüchtlinge aus dem Betonrund. Man folgt den ausgebrannten Wracks an der Stadtautobahn Richtung Osten, wo Kadettenanstalt und Raffinerien die äußersten Ziele waren. Im Herbst kreisen hier die großen Krähenschwärme. Auf einer Gesamtfläche von über achtzig Quadratkilometern bietet unsere Gesellschaft heute eine Investitionsmöglichkeit mit historisch einzigartiger Perspektive. Hier hat die Geschichte dem Anleger voraussetzungslos ein Stück Welt anvertraut, das sich nahezu jungfräulich im Licht der Morgensonne zeigt »

Nach dem Weltuntergang der an die Filmsequenzen aus E Kusturicas " Underground " erinnesrt, formuliert der tote Aschenbrenner dann aus dem Schrank seine Vision für den Weltraum, deren utopischer Gehalt, trotz der Aufgabe der Erde, entfernt an Rinke erinnert : «Um Mitternacht Ortszeit wird die Stadt dann von den westlichen Armeen aufgegeben. Vorübergehend, sagen sie, und sprechen von geregeltem Rückzug, aber die Bilder der Soldaten, die Hubschrauber vom Flugzeugträger ins Meer stürzen, um Platz zu haben für ihre flüchtenden Kameraden, erlauben keinen Zweifel, dass das Gebiet für immer verloren ist. Heute stehen wir vor einer historischen Aufgabe : Die Erschließung von mehreren tausend Quadratkilometern Baugrund in bislang unbewohnter Landschaft. Zunächst werden sich Wissenschaftler um die Bereitstellung von Wasser bemühen, alles weitere, Atmosphäre, Meere, Seen, Flüsse, wird von selbst entstehen, das Leben hat schon einmal aus dem Wasser angefangen. Unter der Einwirkung der veränderten Strahlung werden die Menschen eine Haut aus Gold entwickeln und sich schimmernd durch Städte aus rotem Stein bewegen, denn alles ist dort rot, der ganze Planet, und nicht nur eine Sonne steht am gelben Himmel, man sieht noch andere Gestirne, die dort kreisen in einem großen, gewaltigen Zusammenhang, und in der 
Ferne strahlt die alte Erde und ist so schön und so weit weg. Die Geschichte hat dem Anleger hier voraussetzungs-los ein Stück Welt anvertraut, das sich jungfräulich im Morgenlicht der Himmelskörper zeigt. »

In seinem neuesten Stück Turista (2005) kehrt Mayenburg wieder auf die Erde zurück, wählt dabei aber als Schauplatz ein Campingareal, das in der «Ebene von Waterloo hinter dem Teutoburger Wald an der Marne» liegt, augenfällig den Fluch der kriegerischen Geschichte spiegelt und zudem eine Gesellschaft (Massenkultur) symbolisiert, die in einer «transzendentalen Obdachlosigkeit » auf vermintem Gelände in aller Roheit und Tristesse campiert und die Zeit mit Büchsenbier und dem Essen von « verbranntem Fleisch » totschlägt.

Die Bewohner setzen sich aus auseinandergerissenen, verlogenen kleinbürgerlichen Familien, die erfolglos eine Wiederbelebung proben, gescheiterten Paaren, Jägern sowie einer Gruppe von verhaltensauffälligen Jugendlichen, die in die Gesellschaft reintegriert werden soll, zusammen. Als Themen treten auch hier, im Vergleich zu seinen Familiendramen jedoch in vergrößerter Dimension, wieder Inzest, Missbrauch und Gewalt auf, die Mayenburg in eine milieuunabhängige Kunstsprache montiert.

Eine zentrale dramaturgische Komponente bildet die Figur des jungen Oli, der im Verlauf des Stückes fünf Mal unterschiedlich zu Tode kommt, woraus sich schließen ließe, dass jeder der Campierenden das Potential zum Töten hat und als Täter in Frage kommt. Die Momente des "Schönen", die sich in der Natur (Wald, Fluss) kurz widerspiegeln, sind allerdings durch die historische Vorbelastung des Ortes als auch des möglichen Schauplatzes des Mordes an Oli, doppelt gebrochen. Zudem befindet sich in unmittelbarer Nähe ein Steinbruchgelände, in dem Olis Vater nach abgestorbener Ehe und Familie haust und von dort wiederholt auf das Campingareal vordringt, um seine Ex-Frau mit dessen neuem Partner zu attackieren. Die Gruppe der Jugendlichen probt unter Anleitung eines Sozialarbeiters im Wald nebenan ein Märchen, dessen schöne Züge signifikanter Weise bereits von hässlichen Zügen gebrochen werden und als verspiegeltes Meta-Bild der campierenden Menschen gelesen werden könnte. Das Märchen von «Hänsel und Gretel » erfährt bei Mayenburg eine noch grausamere Spielvariante. Am Ende von Turista, was im Mexikanischen so viel wie "Montezumas Rache" bedeutet, irren die verschiedenen Kinder auf ihrer Suche nach dem Nachhauseweg durch eine Wüste von Steinen. Die Häusersteine sind längst aus dem familiären Mauerwerk gebrochen, die Geschichte radikal gestört und das Weltbild der Erwachsenen aus der Perspektive der Jugendlichen ein einziger Alptraum.

Der tote Oli hat schließlich das letzte Wort : «Wenn ich einmal groß bin, möchte ich klein sein. »

BIBLIOGRAPHIE

Marius von Mayenburg (Vertreten durch den Henschel Schauspiel Verlag, Berlin) : 
Feuergesicht, 1998

Parasiten, 2000

Das kalte Kind, 2002

Eldorado, 2004

Turista, 2005

Moritz Rinke (Vertreten durch den Rowohlt Theater Verlag, Hamburg) :

Republik Vineta, 2000

Die Optimisten, 2003

Café Umberto, 2005

\section{RÉSUMÉS}

In dem Beitrag soll das Bild des Schönen und Hässlichen in der deutschen Gegenwartsdramatik exemplarisch an mehreren Werken von Motitz Rinke und Marius von Mayenburg untersucht werden Ausgegangen wird von der These, dass in Analogie zu Ästhetisierungsprozessen in unserer Gesellschaft, sich der künstlerische Ausdruck des Schönen und Hässlichen in den Dramen zunehmend vermischt und durch die «Ästhetik des Verwischens» nicht mehr in Opposition gelesen werden kann In den Theatertexten lässt sich, so das Ergebnis, eine wachsende Versetzung bzw Zersetzung des Schönen und Hässlichen beobachten, die in ihrem mehrfach gebrochenen dramaturgischen Stadium als Verspiegelung(en) bezeichnet werden sollen.

Nous nous proposons dans cette contribution d'étudier la représentation du beau et du laid dans le théâtre allemand d'aujourd'hui en prenant pour exemple les œuvres de Moritz Rinke et de Marius von Mayenburg. Nous partons pour cela de l'idée que, conformément aux processus d'esthétisation qui s'opèrent dans notre société, la beauté et la laideur, dans leur expression artistique, se confondent de plus en plus, au théâtre, et que, en vertu de l'esthétique de l'effacement, elles ne peuvent plus êtres lues comme des valeurs qui s'opposent On observe ainsi dans les textes de théâtre un déplacement et une désagrégation croissante du beau et du laid, aboutissant au terme des différents stades du travail dramaturgique, à ce que l'on appelle une « réflexion déformante ».

INDEX

Mots-clés : théâtre, beauté, laideur

\section{AUTEUR}

STEFAN TIGGES

Université Rouen/Universität Bremen 